\title{
Continuously tuneable orthogonally polarized RF optical single sideband generator based on integrated micro-ring resonators
}

Yuning Zhang ${ }^{\mathrm{a}}$, Xingyuan $\mathrm{Xu}^{\mathrm{a}}$, Jiayang $\mathrm{Wu}^{\mathrm{a}}$, Linnan Jia ${ }^{\mathrm{a}}$, Mengxi Tan ${ }^{\mathrm{a}}$, Thach G. Nguyen ${ }^{\mathrm{b}}$, Sai T. Chu ${ }^{\mathrm{c}}$, Brent E. Little ${ }^{\mathrm{d}}$, Roberto Morandotti ${ }^{\mathrm{e}, \mathrm{f}, \mathrm{g}}$, Arnan Mitchell ${ }^{\mathrm{b}}$, and David J. Moss ${ }^{\mathrm{a}, *}$

${ }^{a}$ Centre for Micro-Photonics, Swinburne University of Technology, Hawthorn, VIC 3122, Australia

${ }^{\mathrm{b} S}$ School of Engineering, RMIT University, Melbourne, VIC 3001, Australia

cDepartment of Physics and Material Science, City University of Hong Kong, Tat Chee Avenue, Hong Kong, China.

${ }^{\mathrm{d}}$ State Key Laboratory of Transient Optics and Photonics, Xi'an Institute of Optics and Precision Mechanics, Xi'an, China.

' INRS-Énergie, Matériaux et Télécommunications, 1650 Boulevard Lionel-Boulet, Varennes, Québec, J3X 1S2, Canada.

${ }^{f}$ National Research University of Information Technologies, Mechanics and Optics, St. Petersburg, Russia.

${ }^{g}$ Institute of Fundamental and Frontier Sciences, University of Electronic Science and Technology of China, Chengdu,China.

*E-mail: dmoss@swin.edu.au

\begin{abstract}
We demonstrate an orthogonally polarized optical single sideband (OP-OSSB) generator based on dual cascaded micro-ring resonators (MRRs). We achieve a large tuning range of the optical carrier to sideband ratio of up to $57.3 \mathrm{~dB}$. The operation RF frequency of the OP-OSSB generator can also be continuously tuned with a $21.4 \mathrm{GHz}$ range via independent thermal control of the two MRRs.
\end{abstract}

\section{INTRODUCTION}

Photonic radio frequency (RF) signal processing has attracted great interest for a wide range of applications in radar and communications due to its high performance, including broad bandwidth, low loss, high versatility and reconfigurability, and strong immunity to electromagnetic interference [1-3]. In optical communication systems, the modulation formats of optical RF signals directly impact their transmission capacity and spectral efficiency. Offering advantages such as overcoming dispersion-induced distortion, enhanced spectral efficiency, and the ability to separately manipulate the optical carrier and sidebands via polarization-sensitive optical components, orthogonally polarized optical single sideband (OP-OSSB) modulation has been widely exploited in applications ranging from antenna beamforming to microwave photonic signal processing [4]. To achieve OP-OSSB modulation, many approaches have been demonstrated, such as using specially designed modulators and differential group delay elements [5, 6]. However, these approaches face limitations of one form or another, including the intrinsic electronic bandwidth bottleneck, bulky sizes, and stability.

In this paper, we report a continuously tunable (in RF frequency) OP-OSSB generator based on dual cascaded integrated MRRs [7]. The operation RF frequency of the OP-OSSB generator, determined by the spectral interval between the TE and TM resonances, can be dynamically tuned via separate thermo-optical control of the two MRRs, resulting in operation over a wide RF tuning range. Moreover, by controlling the polarization angle of the input light, we achieve a large dynamic tuning range in the optical carrier-to-sideband ratio (OCSR) of up to 57.3 $\mathrm{dB}$. Our device represents a competitive approach towards OP-OSSB generation with wideband tunable operation, and is promising for photonic RF signal transmission and processing in radar and communication systems.

\section{OPERATION PRINCIPLE}

Figure 1(a) shows a schematic of the wideband tunable OP-OSSB generator. Continuous-wave light from a tunable laser source is intensity-modulated by an RF signal to generate a double sideband signal with a polarization angle $\theta$ to the TE-axis (Fig. 1(a-i)), and then fed into the two cascaded MRRs that support TE- and TMpolarization modes. When the wavelength of the optical carrier and the input RF frequency match with the
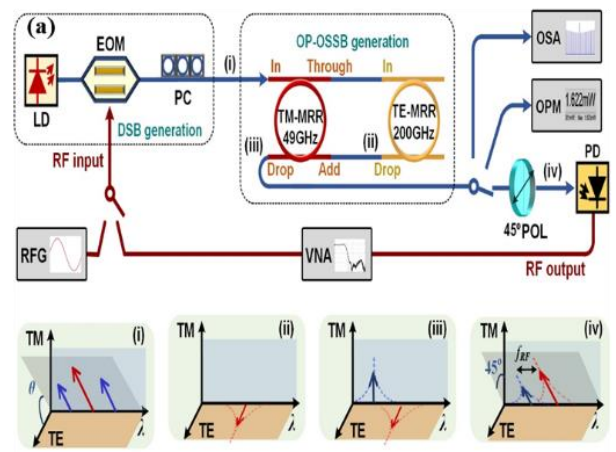

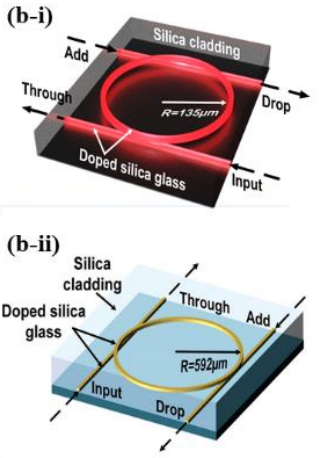

Fig. 1. (a) Schematic of the proposed orthogonally polarized OP-OSSB generator. (b) Schematic illustration of two MRRS . (c) Measured (ci) transmission spectra of the TM-MRR, TE-MRR, and the combined OP-OSSB generator. (c-ii) Zoom-in spectra of (c-i) with one TE polarized resonance and one TM polarized resonance. 
orthogonally polarized resonances of the two MRRs, the optical carrier and one sideband of the double sideband signal can be dropped (Fig. 1(a-ii)). After that, the dropped optical carrier and sideband are combined together by connecting the drop-port of the TE-MRR to the add-port of the TM-MRR (Fig. 1(a-iii)), thus achieving OP-OSSB modulation. The MRRs (with schematic illustration shown in Fig. 1(b)) were fabricated on a high-index doped silica glass platform using CMOS compatible fabrication processes [8-25]. Both MRRs support TE- and TMpolarization modes. The difference between the effective indices of the TE and TM modes resulted in slightly different free spectral ranges (FSRs) for the TE and TM resonances and a wide spectral interval between them in the telecom band. Figure 1 (c) shows the measured transmission spectra of the dual MRRs. The operation RF frequency was determined by the spectral interval between adjacent orthogonally polarized resonances (Fig. 1(cii)). The TM-MRR features a high Q factor, corresponding to a 20dB-bandwidth of $\sim 1.04 \mathrm{GHz}$ (Fig. 1(c-ii)), indicating a high rejection ratio of the optical carrier and lower accessible RF frequency down to the sub-GHz level. Futhermore, we measured the corresponding transmission of the dual MRRs (Fig. 2(a)) as the input polarization angle $(\theta$ in Fig. 1(a-i)) was varied. The extinction ratio between TE and TM resonances varied from $20.5 \mathrm{~dB}$ to $-31.1 \mathrm{~dB}$, corresponding to a dynamic tuning range of up to $51.1 \mathrm{~dB}$ for the OSCR. Figures 2(b)-(c) show the optical spectra of the generated OP-OSSB signal with input RF frequencies at $19.7 \mathrm{GHz}$ and $26.6 \mathrm{GHz}$. Continuously variable OCSRs ranging from -21.1 to $36.2 \mathrm{~dB}$ and from -18.1 to $38.9 \mathrm{~dB}$ were achieved for the 19.7 GHz- and $26.6 \mathrm{GHz}-\mathrm{RF}$ input, respectively, yielding a large OCSR tuning range of up to $57.3 \mathrm{~dB}$.
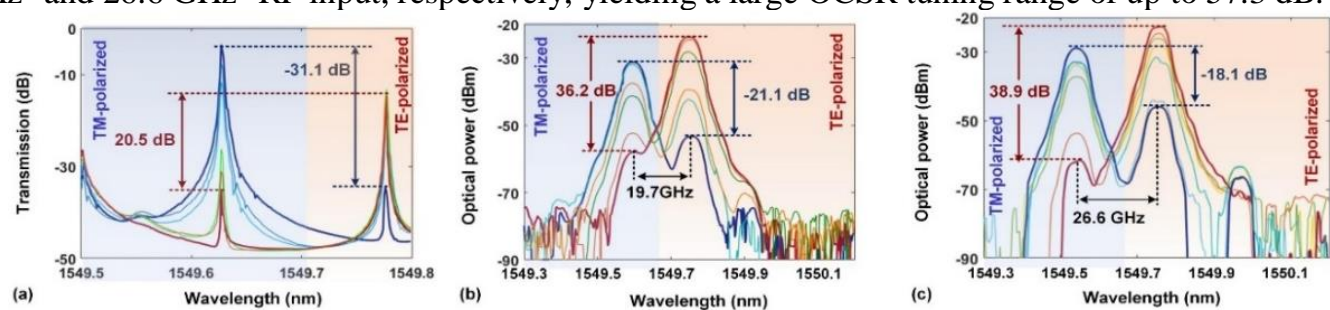

Fig. 2. Measured (a) transmission spectra of the dual MRRs and (b-c) optical spectra of the OP-OSSB signal with continuously tunable OCSR.

To achieve wide RF tunability we varied the spectral interval between the TE and TM resonances by applying independent thermal controls to the TE- and TM-MRRs. Figure 3(a) shows the measured transmission spectra of the dual MRRs as a function of temperature, where the TM-polarized resonance was thermally tuned over a range of $0.2 \mathrm{~nm}$ while the TE-polarized resonance was fixed, thus leading to over $20 \mathrm{GHz} \mathrm{RF}$ tuning range. As shown in Fig. 3(b), wideband RF operation up to $23.14 \mathrm{GHz}$ was demonstrated. The optical spectra of the OP-OSSB signals with tunable RF operation were measured and shown in Fig. 3(c). As the chip temperature of the TMMRR was varied from $22^{\circ} \mathrm{C}$ to $35^{\circ} \mathrm{C}$, the $\mathrm{RF}$ operation frequency changed from $1.81 \mathrm{GHz}$ to $23.27 \mathrm{GHz}$ with a fit slope of $-1.66 \mathrm{GHz} /{ }^{\circ} \mathrm{C}$ (Fig. 3(d)), thus confirming the wide tuning range of our OP-OSSB generator.

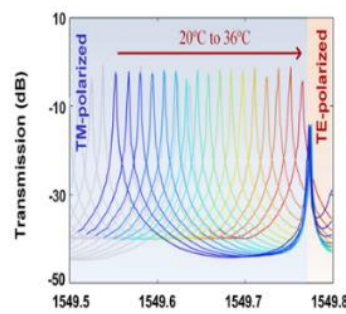

(a) Wavelength $(\mathrm{nm})$

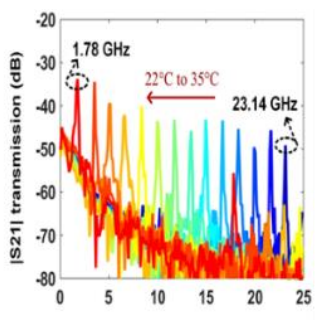

(b)

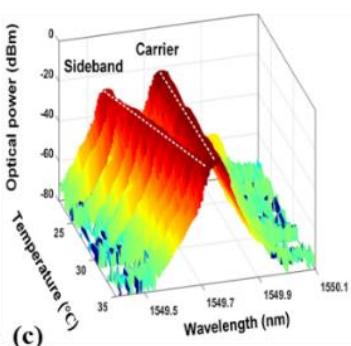

(c)

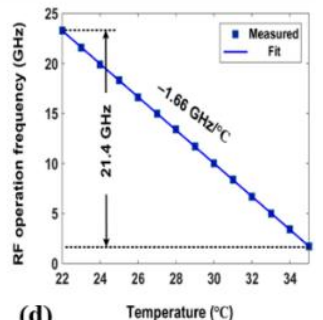

(d)

Fig. 3. Measured (a) optical transmission spectra, and (b) RF transmission response of the proposed OP-OSSB generator with thermo-optical control. (c) Optical spectra and (d) extracted operation RF frequency of the generated OP-OSSB signals with thermal tuning.

\section{REFERENCES}

1. J. Capmany et al., "Microwave photonics combines two worlds," Nat. Photonics, 1(6), 319-330 (2007).

2. J. Wu et.al., "RF photonics: an optical microcombs' perspective," J. Sel. Top. Quantum Electron., 24 (4), 1-20, 2018.

3. J. Wu et.al., "Passive silicon photonic devices for microwave photonic signal processing," Opt. Commun., 373, 2016.

4. X. Xu et.al., "Orthogonally polarized RF optical single sideband generation and dual-channel equalization based on an integrated micro-ring resonator," J. Lightw. Technol., 36(20), 4808-4818, 2018.

5. B. Vidal et.al., "Optical beamforming network based on fiber-optical delay lines and spatial light modulators for large antenna arrays," Photon. Tech. Lett., 18, 2590 (2006).

6. Z. Li et.al., "Linear photonic RF phase shifter using differential-group-delay element and optical phase modulator," Opt. Lett., 35, 1881 (2010).

7. X. Xu et.al., "Continuously tuneable orthogonally polarized RF optical single sideband generator based on micro-ring resonators," J. Opt., 20(11), 115701, 2018.

8. X. Xu et al., "Photonic microwave true time delays for phased array antennas using a 49GHz FSR integrated optical microcomb source," Photonics Res., Vol. 6, No. 5, B30-B36 (2018).

9. X. Xu et.al., "Broadband RF channelizer based on an integrated optical frequency Kerr comb source," J. Lightw. Technol., 36, 4519 (2018).

10. H. Bao et al., "Laser cavity-soliton microcombs," Nature Photonics, vol. 13, no. 6, pp. 384-389, Jun. (2019). 
11. X. Xu et al., "Photonic RF phase-encoded signal generation with a microcomb source", Journal of Lightwave Technology, vol.38, no.7, pp.1722-1727 (2020). DOI:10.1109/JLT.2019.2958564.

12. M. Tan et al., "Photonic RF arbitrary waveform generator based on a soliton crystal micro-comb source", Journal of Lightwave Technology, Early Access (2020). DOI: 10.1109/JLT.2020.3009655.

13. X. Xu et al., "Broadband photonic radio frequency channelizer with 90 channels based on a soliton crystal microcomb", Journal of Lightwave Technology, vol. 38, no. 18, pp. 5116 - 5121 (2020). DOI:10.1109/JLT.2020.2997699.

14. B.eCorcoran et al., "Ultra-dense optical data transmission over standard fiber with a single chip source", Nature Communications, vol. 11, Article: 2568 (2020). DOI:10.1038/s41467-020-16265-x.

15. X. Xu et al., "Photonic RF and microwave integrator with soliton crystal microcombs", IEEE Transactions on Circuits and Systems: Express Briefs, Early Access (2020). DOI:10.1109/TCSII.2020.2995682

16. M.Tan et al., "Photonic RF and microwave filters based on $49 \mathrm{GHz}$ and $200 \mathrm{GHz}$ Kerr microcombs", Optics Communications, vol. 465, Article: 125563 (2020). DOI:10.1016/j.optcom.2020.125563.

17. X. Xu et al., "Photonic perceptron based on a Kerr microcomb for scalable high speed optical neural networks", Laser and Photonics Reviews, vol. 14, no. 8, 2000070 (2020). DOI:10.1002/lpor.202000070.

18. M. Tan et al., "Broadband microwave and RF fractional differentiator using photonics", IEEE Transactions on Circuits and Systems: Express Briefs, Early Access (2020). DOI:10.1109/TCSII.2020.2965158.

19. X. Xu et al., "Broadband microwave frequency conversion based on an integrated optical micro-comb source", Journal of Lightwave Technology vol. 38, no. 2, pp.332-338 (2020). DOI: 10.1109/JLT.2019.2930466

20. M. Tan et al., "Broadband microwave and RF photonic fractional Hilbert transformer based on a 50GHz integrated Kerr micro-comb", Journal of Lightwave Technology vol.37, no.24, pp. 6097 - 6104 (2019). DOI: 10.1109/JLT.2019.2946606.

21. Xingyuan Xu, Mengxi Tan, Jiayang Wu, Roberto Morandotti, Arnan Mitchell, and David J. Moss, "Microcomb-based photonic RF signal processing", IEEE Photonics Technology Letters 31 (23) 1854-1857 (2019). DOI: 10.1109/LPT.2019.2940497

22. X. Xu et al., "Advanced adaptive photonic RF filters based on an optical micro-comb source with 80 taps", Journal of Lightwave Technology, vol. 37, no. 4, pp. 1288-1295 (2019). DOI:10.1109/JLT.2019.2892158

23. X. Xu et al., "High performance RF filters via bandwidth scaling with Kerr micro-combs", Applied Physics Letters Photonics vol.4, 026102 (2019); doi:10.1063/1.5080246.

24. J. Wu et al., "RF photonics: An optical micro-combs' perspective", IEEE Journal of Selected Topics in Quantum Electronics 24 (4), 1-20, Article: 6101020 (2018). DOI: 10.1109/JSTQE.2018.2805814.

25. X. Xu et al., "Advanced RF and microwave functions based on an integrated optical frequency comb source", Optics Express vol.26, no. 3, 2569-2583 (2018). DOI:10.1364/OE.26.002569 